\title{
Muscle-driven and torque-driven centrodes during modeled flexion of individual lumbar spines are disparate
}

\author{
Robert Rockenfeller ${ }^{1}\left[\right.$ (D) Andreas Müller ${ }^{2,3} \cdot$ Nicolas Damm $^{2} \cdot$ Michael Kosterhon $^{4}$ - Sven R. Kantelhardt ${ }^{4}$. \\ Rolfdieter Frank ${ }^{1} \cdot$ Karin Gruber ${ }^{2}$
}

Received: 6 March 2020 / Accepted: 24 August 2020 / Published online: 16 September 2020

(c) The Author(s) 2020

\begin{abstract}
Lumbar spine biomechanics during the forward-bending of the upper body (flexion) are well investigated by both in vivo and in vitro experiments. In both cases, the experimentally observed relative motion of vertebral bodies can be used to calculate the instantaneous center of rotation (ICR). The timely evolution of the ICR, the centrode, is widely utilized for validating computer models and is thought to serve as a criterion for distinguishing healthy and degenerative motion patterns. While in vivo motion can be induced by physiological active structures (muscles), in vitro spinal segments have to be driven by external torque-applying equipment such as spine testers. It is implicitly assumed that muscle-driven and torque-driven centrodes are similar. Here, however, we show that centrodes qualitatively depend on the impetus. Distinction is achieved by introducing confidence regions (ellipses) that comprise centrodes of seven individual multi-body simulation models, performing flexion with and without preload. Muscle-driven centrodes were generally directed superior-anterior and tail-shaped, while torque-driven centrodes were located in a comparably narrow region close to the center of mass of the caudal vertebrae. We thus argue that centrodes resulting from different experimental conditions ought to be compared with caution. Finally, the applicability of our method regarding the analysis of clinical syndromes and the assessment of surgical methods is discussed.
\end{abstract}

Keywords Axis of rotation $\cdot$ Finite helical axis $\cdot$ Confidence ellipse $\cdot$ Biomechanics $\cdot$ Spine model $\cdot$ Hill-type muscle model

\section{Introduction}

Electronic supplementary material The online version of this article (https://doi.org/10.1007/s10237-020-01382-9) contains supplementary material, which is available to authorized users.

Robert Rockenfeller

rrockenfeller@uni-koblenz.de

1 Mathematical Institute, University Koblenz-Landau, Universitätsstr. 1, 56070 Koblenz, Germany

2 Institute for Medical Engineering and Information Processing (MTI Mittelrhein), University Koblenz-Landau, Universitätsstr. 1, 56070 Koblenz, Germany

3 Mechanical Systems Engineering Laboratory, EMPA-Swiss Federal Laboratories for Materials Science and Technology, Ueberlandstr. 129, 8600 Dübendorf, Switzerland

4 Department of Neurosurgery, University Medical Centre, Johannes Gutenberg-University, Langenbeckstr. 1, 55131 Mainz, Germany
Relative movement between (lumbar) vertebrae occurs during most daily motions. In three dimensions, such a relative movement between two time instances can be represented by a finite helical (or screw) axis (FHA), i.e., an (instantaneous) axis of rotation that points toward the direction of possible translations. By intersecting the FHA with an anatomical plane, an instantaneous center of rotation (ICR) is obtained. The time evolution (path) of the ICR, the socalled centrode, had been investigated by multiple researchers with highly diverse and ambitious objectives, e.g. (1) recognizing general patterns in order to "give base-line references for potential diagnostic applications" (Aiyangar et al. 2017), "predicting...injurious vectors" (Qiu et al. 2003), and finding an "indicator for mechanical disorders" (Schmidt et al. 2008) or "motion characteristics of the normal lumbar spine" (Yoshioka et al. 1990), (2) evaluating "the quality, rather than the quantity, of cervical spine movement" (Baillargeon and Anderst 2013), (3) hoping for the ICR to be "interpreted in terms of...anatomical and pathological 
factors" (Bogduk et al. 1995), (4) describing the change in ICR location as a consequence of disk degeneration (Cossette et al. 1971; Ellingson and Nuckley 2015; Gertzbein et al. 1985), (5) attempting to relate the ICR location to the "choice of anterior and posterior instrumentation" (Haher et al. 1991) or certain implant parameters (Niosi et al. 2006), (6) demonstrating that "analysis for sagittal plane motion of the lumbar spine is possible" (Ogston et al. 1986), and (7) correlating ICR paths to facet forces (Rousseau et al. 2006). However, a recent review (Widmer et al. 2019) had revealed that, up to now, the ICR provides only faint criteria for the description of spinal kinematics under healthy and degenerative conditions.

In this work, we used elementary, individualized multibody simulation (MBS) models of the lumbar spine (Damm et al. 2019), performing flexion movements, to introduce a statistical criterion that may serve as a first step toward describing, detecting, and eventually understanding the cause and effect of the centrode's location: the (weighted) confidence ellipse as introduced in Sect. 2.4. The underlying assumption was that similar (individual) spinal structures, under the same loading conditions, yield a similar relative motion of segments. Of course, changes in the individual geometries cause different relative motion patterns and thus different centrodes. Yet, we show that these differences were small under the same loading condition, but distinguishable under varying conditions. Our method was utilized to identify centrode locations depending on (1) individual (a) Neutral position, lateral view

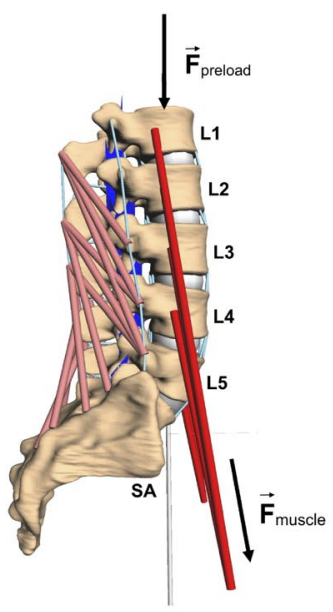

(b) Neutral position, lateral view

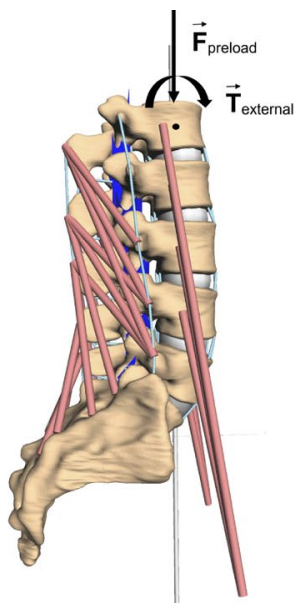

Fig. 1 Example of an individual spine model (L1-SA) with vertebral surfaces extracted from DICOM data. Ligament (light blue lines) and muscle (red lines) insertion points were set at typical anatomic landmarks. Ligaments, intervertebral disks (gray ellipsoids) and facet joints (blue planes) transmit forces. Passive muscles are depicted in a paler red tone, whereas active muscles were depicted in vibrant red. The line of action of the preload force, representing the upper body geometries, (2) force application modes, and (3) material properties that are influenced by clinical syndromes. First, the location of the centrode across individual spine geometries was calculated, both with and without preload representing the upper body weight. This individualized modeling is particularly worthwhile, since, on the one side, modelers from MBS and finite element (FE) communities usually employ generic models (Abouhossein et al. 2013; Qiu et al. 2003; Senteler et al. 2017; Schmidt et al. 2008), which cannot account for structure-based deviations. Experimenters, on the other side, conduct helpful individual measurements, but have no elaborated model on hand (Cossette et al. 1971; Gertzbein et al. 1985; Haher et al. 1991; Niosi et al. 2006; Ogston et al. 1986). Second, the differences in conducting physiologically-based (muscle-driven) and artificial (torquedriven) movement on the centrode were investigated, likewise with and without preload. Third, our method could aid to assess the influence of clinical syndromes or treatments on the centrode's location, as is discussed on the example of modeling the surgical fixation of vertebrae.

\section{Model and methods}

\subsection{Model}

In total, seven individual lumbar spine models (L1-SA, cf. Fig. 1) were investigated. The term 'individual' here (c) Neutral position, ventral view

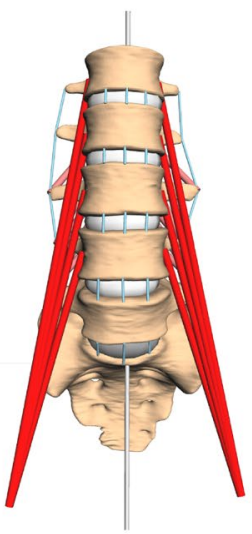

(d) Flexed position, lateral view weight, was located between the femoral heads (gravity line, silver). a, b The neutral position in lateral view, together with the acting forces $\left(F_{\text {preload }}, F_{\text {muscle }}\right)$ and an external torque $\left(T_{\text {external }}\right)$, respectively. c Depicts the corresponding ventral view. Slight asymmetries, resulting from individual geometries can be detected, see also Table 1. d A fully flexed spine resulting from applying muscle force and preload (PM), see Sects. 2.1-2.3 
refers to the vertebral surfaces, including inter-vertebral distances, and ligament insertion points that were extracted from digital image data (DICOM). Geometries of healthy patients (six male and one female with an age of $32.6 \pm 7.04$ years) were provided by the university clinic in Mainz. After semi-automatic segmentation, vertebral surfaces were loaded into a MBS tool (SIMPACK: Dassault Systèmes, Vélizy-Villacoublay, France) under preservation of in vivo distances for intervertebral disks and facet joints. Ligament insertion points were set manually (Schünke et al. 2015) and counter-checked by neurosurgeons from the university clinic in Mainz. All passive force-transmitting structures, i.e., intervertebral disks (IVD), ligaments and facet joints, were modeled as nonlinear spring-damper elements (Damm et al. 2019). Here, particularly force-length and force-angle characteristics of ligaments and IVD, respectively, were extracted from step-wise reduction experiments (Heuer et al. 2007) and validated using data on range of motion (Heuer et al. 2007) as well as intradiscal pressure (Wilke et al. 1996). Ligaments were found to be significantly less stiff than suggested by classical data sets, e.g. (Chazal et al. 1985; Shirazi-Adl et al. 1986; White and Panjabi 1990). Indeed, recent work, combining in vivo experiments and computer simulation, support this finding (Mörl et al. 2020). Ligament pre-strain was set according to literature data, cf. (Damm et al. 2019, sect. 2.2.4).
Active forces were transmitted by Hill-type muscle models (Guenther et al. 2007; Haeufle et al. 2014; Rockenfeller and Guenther 2016, 2018) of M. psoas major and M. multifidus, each strand modeled as a one-dimensional point-topoint element with no deflection. Since neither kinematic nor kinetic data were available to conduct an elaborated parameter estimation, tendon and fiber lengths parameters were taken from literature, particularly (Christophy et al. 2012). For every muscle strand, values for optimal fiber length and tendon slack length were adapted from their Table 1, columns seven and nine, respectively. In order to maintain the fiber-to-tendon length ratio as an important functional measure (Mörl et al. 2015), both quantities were equally scaled to match individual geometries, e.g. each increased by $5 \%$ if the distance from origin to insertion was $5 \%$ higher than the literature reference. The fiber-to-tendon length ratios for each muscle strand were 2.85 for M. psoas and 2.47 for M. multifidus. For one model, exemplary optimal fiber lengths and tendon slack lengths for both muscle groups are displayed in Table 1. M. psoas maximum force was set to $80 \mathrm{~N}$ for each strand, the median value from Christophyet al. (Christophy et al. 2012, table 1, fourth column) for non-IVD parts. M. multifidus force was set to $21 \mathrm{~N}$ for each strand, likewise the median value for non-laminar parts. Remaining parameters of the muscle model were assumed to be generic and taken from (Guenther et al. 2007, table 2)

Table 1 Optimal fiber lengths (OFL) and tendon slack lengths (TSL) of each strand within the M. psoas and M. multifidus group, exemplary for one lumbar spine model

\begin{tabular}{|c|c|c|c|c|c|c|c|c|c|c|}
\hline \multicolumn{11}{|l|}{ Muscle group } \\
\hline \multicolumn{11}{|l|}{ M. psoas } \\
\hline Strand & Ps_L1 & Ps_L2 & Ps_L3 & Ps_L4 & & & & & & \\
\hline \multicolumn{11}{|l|}{ Sinister } \\
\hline OFL (m) & 0.212 & 0.184 & 0.156 & 0.127 & & & & & & \\
\hline TSL (m) & 0.0744 & 0.0647 & 0.0549 & 0.0448 & & & & & & \\
\hline \multicolumn{11}{|l|}{ Dexter } \\
\hline OFL (m) & 0.217 & 0.189 & 0.160 & 0.130 & & & & & & \\
\hline TSL (m) & 0.0763 & 0.0336 & 0.0561 & 0.0456 & & & & & & \\
\hline Ratio & 2.85 & & & & & & & & & \\
\hline \multicolumn{11}{|l|}{ M. multifidus } \\
\hline Strand & Mf_SA_L5 & Mf_SA_L4 & Mf_SA_L3 & Mf_SA_L2 & Mf_L5_L3 & M_L5_L2 & Mf_L5_L1 & Mf_L4_L2 & Mf_L4_L1 & Mf_L3_L1 \\
\hline \multicolumn{11}{|l|}{ Sinister } \\
\hline OFL (m) & 0.0504 & 0.0519 & 0.0668 & 0.0864 & 0.0626 & 0.0830 & 0.107 & 0.0615 & 0.0837 & 0.0655 \\
\hline TSL (m) & 0.0204 & 0.0210 & 0.0270 & 0.0350 & 0.0253 & 0.0336 & 0.0434 & 0.0249 & 0.0339 & 0.0265 \\
\hline \multicolumn{11}{|l|}{ Dexter } \\
\hline OFL (m) & 0.0523 & 0.0537 & 0.0688 & 0.0881 & 0.0644 & 0.0852 & 0.109 & 0.0652 & 0.0864 & 0.0661 \\
\hline TSL (m) & 0.0212 & 0.0217 & 0.0278 & 0.0358 & 0.0261 & 0.0345 & 0.0442 & 0.0264 & 0.0350 & 0.0268 \\
\hline Ratio & 2.47 & & & & & & & & & \\
\hline
\end{tabular}

Strands from M. psoas (Ps) are labeled with the corresponding insertion point at the lumbar spine, whereas strands of the M. multifidus (Mf) are labeled with both origin and insertion, cf. Fig. 1. The listing serves the purpose of estimating the magnitudes of each muscle strand as well as demonstrating the slight asymmetry of sinister and dexter geometries. The fiber-to-tendon length ratios (OFL/TSL) for each group, which were applied for all seven models, are also given 
(contraction dynamics) and (Rockenfeller and Guenther 2018) (activation dynamics).

\subsection{Calculating the instantaneous FHA and ICR during flexion}

For comparability, we hereinafter investigated only flexion of the modeled lumbar spines, i.e., the movement that occurs during forward bending of the upper body, see Fig. 1d and the supplementary video file. This flexion was driven either by muscle forces or an external torque applied at the COM of vertebra L1 (Abouhossein et al. 2013), see also next Sect. 2.3. We determined the finite helical axis (FHA) between two vertebrae using a least-squares method on spatial coordinates (Kwon 2008; Spoor and Veldpaus 1980). Therefore, four markers (ligament insertion points of ligamentum supraspinale, flavum sinister, intertransversale sinister and anterior longitudinal dexter) on each vertebral body were tracked at each time instance (every millisecond), relative to the subjacent vertebra. In a subsequent step, the intersection point of the FHA with the corresponding anatomical plane-in our case the sagittal plane-was calculated and defined to be the instantaneous center of rotation (ICR). Figure 2 depicts the situation for an exemplary L4-L5 segment. The reduction to a two-dimensional quantity is reasonable here, because the observed motion (flexion) only takes place in the sagittal plane itself. This means the vertebrae can be assumed to undergo no substantial relative translation along the helical axis, which ought to be approximately perpendicular to the sagittal plane, and thus perform a pure rotation. We further note: First, the term 'instantaneous' is rather to be read as 'approximately instantaneous' or 'finite', because no rigorous differential-geometric approach has been applied. However, observed time intervals were small and thus this term was chosen in order to retain consistency with the literature. Second, most in vivo/vitro studies (Bogduk et al. 1995; Cossette et al. 1971; Haher et al. 1991; Yoshioka et al. 1990) and even elaborated FE models (Qiu et al. 2003; Shirazi-Adl et al. 1986; Schmidt et al. 2008) calculate only elementary, two-dimensional rotation with Reuleaux' method (Reuleaux 1875), which is neither applicable in arbitrary three-dimensional motion nor captures translational movement of vertebrae.

\subsection{Simulation protocol: muscle-driven and torque-driven flexion}

In order to investigate the differences in the paths of the ICR, when either driven by muscle (psoas) forces or $10 \mathrm{Nm}$ torque, we defined four different simulation scenarios of lumbar spine flexion. Two scenarios with and two without $500 \mathrm{~N}$ preload, representing the upper body weight: (1) no preload and muscle-driven (NPM), (2) preload and muscle-driven (PM), (3) no preload and torque-driven (NPT), (4) preload and torquedriven (PT). Pre-load forces were supposed to act on L1 and along the gravity line (Le Huec et al. 2011), i.e., the line that vertically bisects the two femoral heads and is thought to go through the center of gravity of the upper body, cf. Fig. 1.

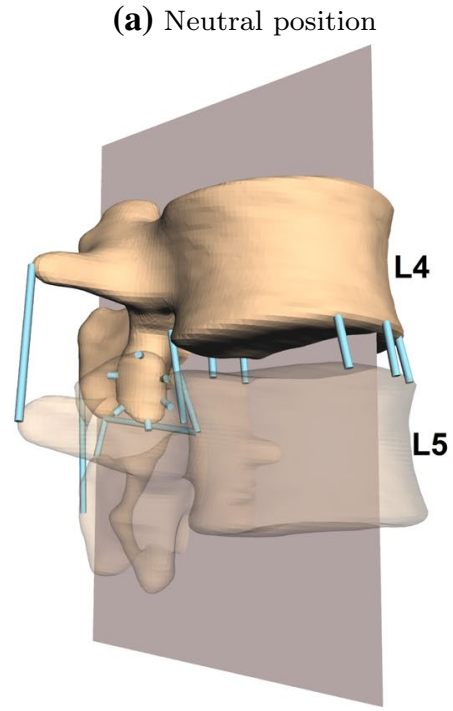

Fig. 2 Exemplary depiction of a centrode resulting from the motion (flexion) of the vertebra L4 relative to L5. This figure is of illustrative character only. For simulated centrodes, see Figs. 3 and 4. For an animated flexion of the whole lumbar spine see the supplementary video file. a Neutral position of the two vertebrae L4-L5 (cf. Fig. 1a) without muscles and disk; vertebral bodies (the upper solid, the lower (b) Flexed position

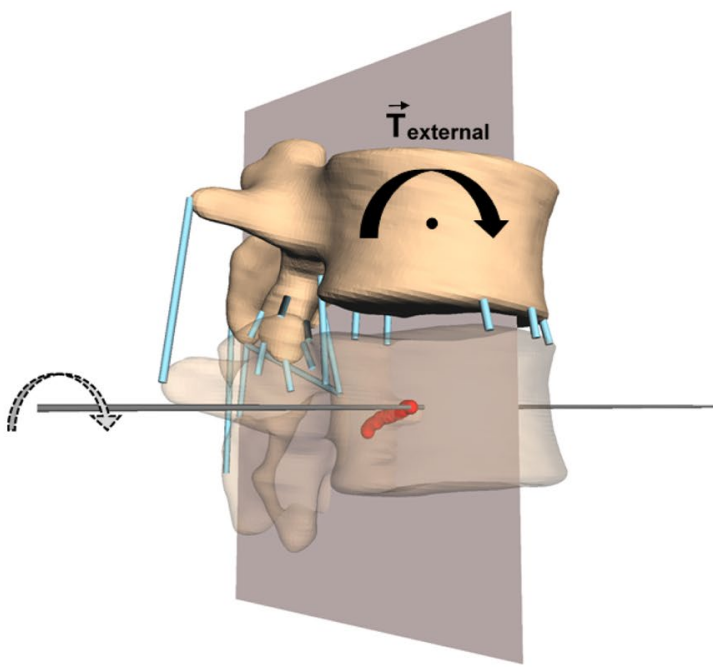

transparent), ligaments (blue strands) and sagittal plane (reddish gray) are depicted. b Flexed position of the same two vertebral bodies at the end of the movement. Besides the structures from a, the FHA at the final time instance (black line) as well as the intersections points of all FHA with the sagittal plane (centrode, red dots), are shown 
Since CT data were obtained from decumbent patients, those scenarios were supposed to represent lying (unloaded) and standing (preloaded) positions. Torque was applied on the COM of the upmost vertebral body (L1). Total simulation time was $2.5 \mathrm{~s}$ in each scenario for each spine. M. psoas major started in a fully activated equilibrium $(q \approx 1)$ and was fully stimulated $(\sigma=1)$ for the whole simulation period, for the notation see (Rockenfeller and Guenther 2018). M. multifidus was neither activated ( $\left.q=q_{0} \approx 0.01\right)$ nor stimulated $(\sigma=0)$ and therefore had only passive, antagonistic contributions on the ICR paths in all cases, cf. also (Zwambag,Brown 2020). Although the high stimulation value for M. psoas major, is far from physiological reality, it was chosen (a) for comparability of all simulation outputs and (b) to obtain a significant motion at all with this reduced model, see also the discussion.

\subsection{Confidence ellipses for the instantaneous center of rotation}

To quantify the effects of individual geometry on ICR paths, we calculated a $95 \%$ confidence ellipse for each pair of adjacent vertebra in each of the afore-defined scenarios. The center point of the ellipses was calculated as the mean of the twodimensional path data, and the semi-axes were represented by the two eigenvectors of the respective covariance matrix (Draper 1998; Galton 1886; Spruyt 2014). The lengths of the semi-axes thus indicate the corresponding standard deviations. We noted that variations in movement of the vertebrae were larger at the beginning than at the end of the $2.5 \mathrm{~s}$ simulation, because the flexed equilibrium had been reached. To account for that fact, we additionally calculated exponentially weighted confidence ellipses of the $n$ (in our case $n=2500$ ) data points by the discrete mapping $w_{\alpha, n}:\{1, \ldots, n\} \rightarrow[0,1]$, with

$$
\begin{aligned}
w_{\alpha, n}(k) & =\frac{\exp ((n-k) \cdot \alpha)}{\sum_{l=1}^{n} \exp ((n-l) \cdot \alpha)} \\
& =\frac{\exp ((n-k) \cdot \alpha) \cdot(\exp (\alpha)-1)}{\exp (n \cdot \alpha)-1} .
\end{aligned}
$$

Weighted mean $\left(\overline{\mathbf{x}}_{w}\right)$ and covariance matrix $\left(C_{w}\right)$ of the twodimensional data $\mathbf{x}=\left\{\left(x_{1}, y_{1}\right)^{T}, \ldots,\left(x_{n}, y_{n}\right)^{T}\right\}$ were calculated by

$\overline{\mathbf{x}}_{w}=\left(\begin{array}{l}\bar{x} \\ \bar{y}\end{array}\right)=\sum_{k=1}^{n} w_{\alpha, n}(k) \cdot\left(\begin{array}{l}x_{k} \\ y_{k}\end{array}\right)$

and

$C_{w}=\sum_{k=1}^{n} w_{\alpha, n}(k) \cdot\left(\begin{array}{ll}\left(x_{k}-\bar{x}\right) \cdot\left(x_{k}-\bar{x}\right) & \left(x_{k}-\bar{x}\right) \cdot\left(y_{k}-\bar{y}\right) \\ \left(x_{k}-\bar{x}\right) \cdot\left(y_{k}-\bar{y}\right) & \left(y_{k}-\bar{y}\right) \cdot\left(y_{k}-\bar{y}\right)\end{array}\right)$.

Here, $\alpha=10 / n$ was found to be a suitable compromise between the location and the narrowness of the final confidence ellipses. The larger $\alpha$ the more weight is attributed to the early data points. In the non-weighted case, $w_{\alpha, n}(k)=1 / n$ for all $k \in\{1, \ldots, n\}$.

\section{Results}

Figure 3 shows the ICR-time courses between all pairs (levels) of adjacent vertebrae (L1-L2 to L5-SA) for seven individual lumbar spine MBS models during flexion. The global coordinates for the mean centers of mass were: $\mathrm{COM}_{\mathrm{L} 3}=(25.8,119.6), \mathrm{COM}_{\mathrm{L} 4}=(32.5,83.4)$, $\mathrm{COM}_{\mathrm{L} 5}=(31.2,48.5)$, and $\mathrm{COM}_{\mathrm{SA}}=(0,0)$ (units: $\left.[\mathrm{mm}]\right)$. ICR coordinates were calculated relative to the initial positions of the centers of mass (COM) of the caudal vertebrae, which were graphically overlayed for all seven spines for easier comparison. Consequently, a 'mean' (out of seven) lumbar spine is shown in the background of each sub-figure, not representing a real lumbar spine but constituting for visual assistance. Confidence ellipses for the non-weighted and the weighted case on each level indicate the location of $95 \%$ of all ICR coordinates across all simulated models. Table 2 provides center locations, semi-axes lengths, and orientation angle of all ellipses as well as obtained range of motion (ROM) of the corresponding lumbar spines. The following paragraphs contain a more detailed description of the four herein investigated load scenarios, i.e., PM, NPM, PT, NPT, see Sect. 2.3.

NPM Fig. 3a shows the ICR-time courses-and corresponding confidence ellipses for each level of the lumbar spines- that resulted from simulating the muscle-driven scenario without any preload (NPM). In each level of the individual, muscle-driven lumbar spine models, the ICR-time courses showed similar behavior: starting inferior-posterior to the center of mass of the caudal vertebra and moving in a superior-anterior direction. The first semi-axis lengths of the corresponding (non-weighted) ellipses increased with caudal position and were significantly longer (up to factor 5) than the second semi-axis lengths, cf. Table 2. Noticeably, the orientation angle of these ellipses did not vary substantially across spine levels $\left(32^{\circ}-37^{\circ}\right)$. The lower the spine level (L1-L2 to L5-SA), the more ellipse centers were found to move in superior-anterior direction, with the center of the L3-L4 ellipse almost congruent to the L4 COM. Introducing the weight function (Eq. (1)) for the purpose of including early ICR data, resulted in an increase in orientation angle $\left(33^{\circ}-50^{\circ}\right)$ as well as an increase particularly in first semi-axis lengths. ROM values from $5.1^{\circ}$ to $14.5^{\circ}$ were found comparable to the NPT scenario.

NPT Fig. 3b shows the ICR-time courses that resulted from simulating the torque-driven scenario without any preload (NPT). In any level of the torque-driven spine models, with exception of L5-SA, the ICR-time courses 
Fig. 3 Two-dimensional locations of the instantaneous center of rotation (ICR) over time (colored dots), obtained from flexion movement of seven individual lumbar spine models. Coordinates at each instant of time were computed by intersecting the finite helical axis (FHA) with the sagittal plane. ICR coordinates were obtained relative to the center of mass $(\mathrm{COM})$ of the caudal vertebra. Colors represent the spinal level (blue: L1-L2, red: L2-L3, yellow: L3-L4, violet: L4-L5, green: L5-SA). For comparability, the COMs of all seven spines at each level were superimposed (red dots). Supportive transparent vertebral surfaces are supplied in the background. These surfaces do not represent a fully physiological lumbar spine, but constitute for averaged constellations. Two types of $95 \%$ confidence ellipses for each level are shown: classical, non-weighted ellipses (solid black lines) and weighted ellipses (dotted black lines, see Eq. (1)) to capture variations in early movement. For ellipse parameters, see Table 2 (a) NPM flexion

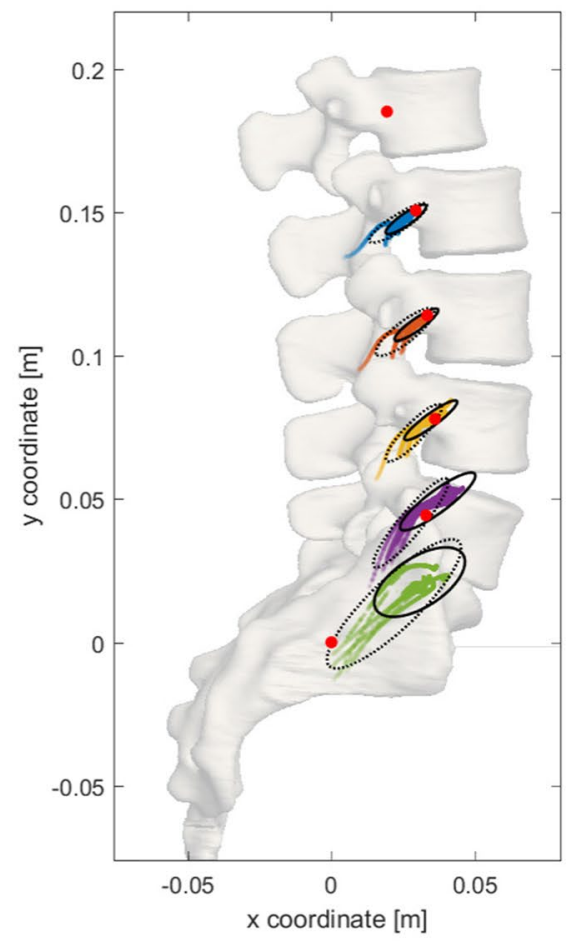

(c) PM flexion

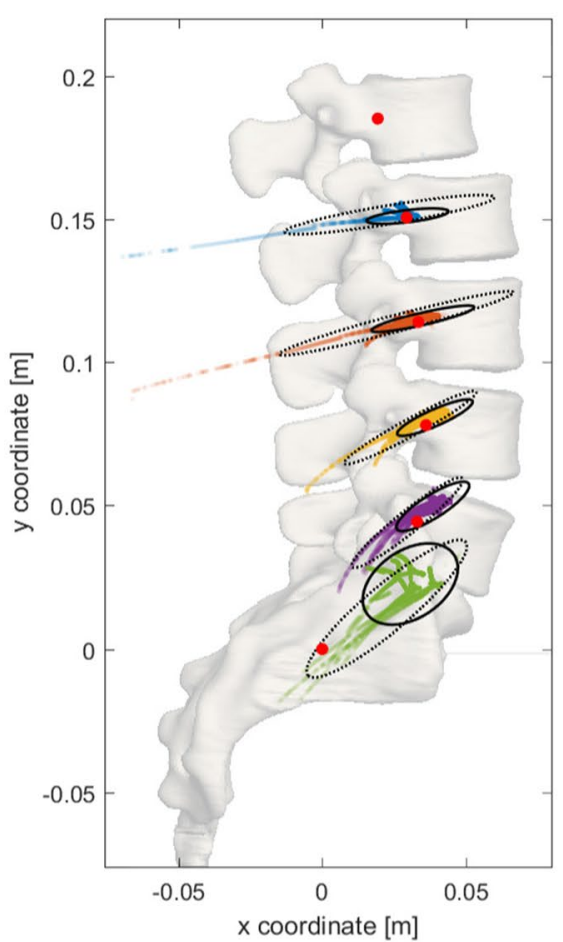

(b) NPT flexion

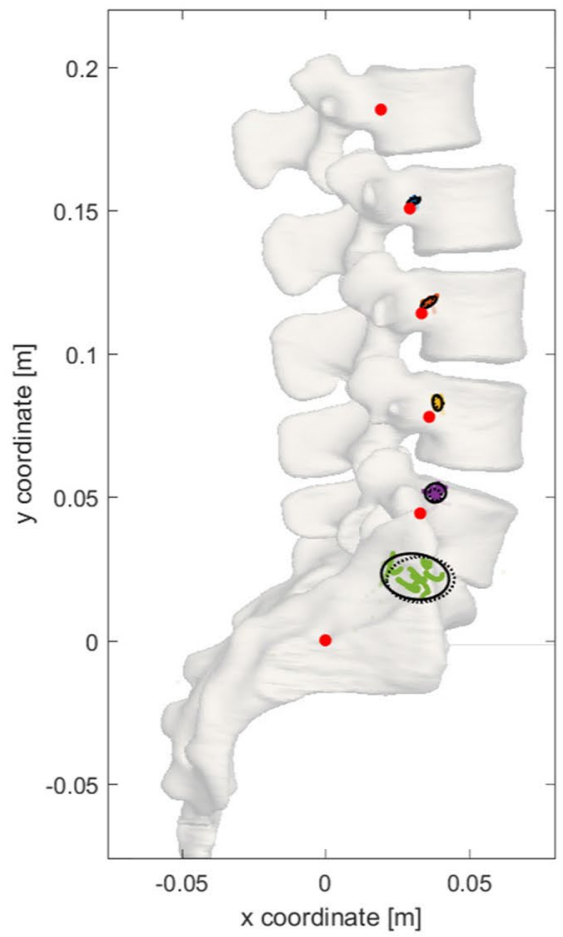

(d) PT flexion

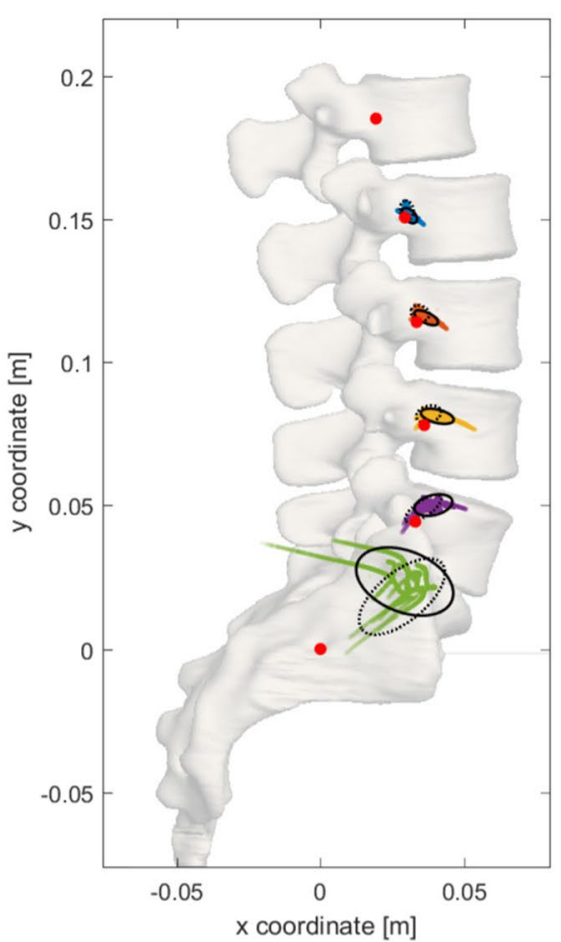

exhibited a similar behavior: for the whole time horizon of simulation, the ICR was located in a narrow region, superior-anterior and close to the COM of the caudal vertebra. Consequently, the semi-axis lengths were distinctly shorter than in the muscle-driven scenarios and the quotients between the first and second semi-axis lengths were substantially smaller, cf. Table 2. Eventually, this quotients became approximately 1 on the L4-L5 level, transforming the ellipses almost into circles. Contrary to the muscle-driven scenarios, in the NPT scenario the weighted ellipses were even 
Table 2 ICR confidence ellipse parameters for muscle- and torque-driven flexion scenarios with and without preload (column one: NPM, NPT, PM, PT) as displayed in Fig. 3

\begin{tabular}{|c|c|c|c|c|c|c|c|c|}
\hline \multirow[t]{2}{*}{ Scenario } & \multirow{2}{*}{$\begin{array}{l}\mathrm{ROM} \\
\text { mean } \pm \mathrm{SD} \\
{[\min \ldots \max ]}\end{array}$} & \multirow[t]{2}{*}{ Level } & \multicolumn{3}{|c|}{ Non-weighted ellipse } & \multicolumn{3}{|c|}{ Weighted ellipse } \\
\hline & & & $\begin{array}{l}\text { Ellipse } \\
\text { center }[\mathrm{mm}]\end{array}$ & $\begin{array}{l}\text { Semi-axes } \\
\text { length [mm] }\end{array}$ & $\begin{array}{l}\text { Orientation } \\
\text { angle }\end{array}$ & $\begin{array}{l}\text { Ellipse } \\
\text { center }[\mathrm{mm}]\end{array}$ & $\begin{array}{l}\text { Semi-axes } \\
\text { length [mm] }\end{array}$ & $\begin{array}{l}\text { Orientation } \\
\text { angle }\end{array}$ \\
\hline \multirow[t]{5}{*}{ NPM } & \multirow[t]{5}{*}{$\begin{array}{l}8.2^{\circ} \pm 3.0^{\circ} \\
{\left[5.1^{\circ} \ldots 14.5^{\circ}\right]}\end{array}$} & L1-L2 & $\left(\begin{array}{c}26.0 \\
147.5\end{array}\right)$ & $\begin{array}{l}a=8.5 \\
b=2.1\end{array}$ & $35^{\circ}$ & $\left(\begin{array}{c}23.3 \\
146.2\end{array}\right)$ & $\begin{array}{l}a=11.9 \\
b=2.8\end{array}$ & $33^{\circ}$ \\
\hline & & L2-L3 & $\left(\begin{array}{c}29.7 \\
110.7\end{array}\right)$ & $\begin{array}{l}a=9.3 \\
b=2.1\end{array}$ & $35^{\circ}$ & $\left(\begin{array}{c}25.8 \\
108.2\end{array}\right)$ & $\begin{array}{l}a=12.7 \\
b=3.6\end{array}$ & $38^{\circ}$ \\
\hline & & L3-L4 & $\left(\begin{array}{l}34.7 \\
77.5\end{array}\right)$ & $\begin{array}{l}a=11.4 \\
b=2.2\end{array}$ & $36^{\circ}$ & $\left(\begin{array}{l}28.8 \\
73.1\end{array}\right)$ & $\begin{array}{l}a=13.6 \\
b=3.4\end{array}$ & $46^{\circ}$ \\
\hline & & L4-L5 & $\left(\begin{array}{l}37.1 \\
49.2\end{array}\right)$ & $\begin{array}{l}a=16.2 \\
b=3.8\end{array}$ & $37^{\circ}$ & $\left(\begin{array}{l}27.8 \\
41.7\end{array}\right)$ & $\begin{array}{l}a=20.0 \\
b=4.1\end{array}$ & $50^{\circ}$ \\
\hline & & L5-SA & $\left(\begin{array}{l}30.8 \\
21.0\end{array}\right)$ & $\begin{array}{l}a=18.0 \\
b=8.7\end{array}$ & $32^{\circ}$ & $\left(\begin{array}{l}21.9 \\
13.4\end{array}\right)$ & $\begin{array}{l}a=31.4 \\
b=8.6\end{array}$ & $44^{\circ}$ \\
\hline \multirow[t]{5}{*}{ NPT } & \multirow[t]{5}{*}{$\begin{array}{l}10.7^{\circ} \pm 1.2^{\circ} \\
{\left[9.1^{\circ} \ldots 12.9^{\circ}\right]}\end{array}$} & L1-L2 & $\left(\begin{array}{c}30.8 \\
153.0\end{array}\right)$ & $\begin{array}{l}a=2.3 \\
b=0.9\end{array}$ & $21^{\circ}$ & $\left(\begin{array}{c}30.9 \\
153.6\end{array}\right)$ & $\begin{array}{l}a=2.1 \\
b=0.9\end{array}$ & $20^{\circ}$ \\
\hline & & L2-L3 & $\left(\begin{array}{c}36.1 \\
117.8\end{array}\right)$ & $\begin{array}{l}a=3.1 \\
b=1.3\end{array}$ & $33^{\circ}$ & $\left(\begin{array}{c}35.9 \\
117.9\end{array}\right)$ & $\begin{array}{l}a=1.8 \\
b=1.1\end{array}$ & $18^{\circ}$ \\
\hline & & L3-L4 & $\left(\begin{array}{l}39.1 \\
82.9\end{array}\right)$ & $\begin{array}{l}a=2.7 \\
b=1.6\end{array}$ & $-83^{\circ}$ & $\left(\begin{array}{l}39.4 \\
83.0\end{array}\right)$ & $\begin{array}{l}a=1.9 \\
b=1.6\end{array}$ & $-42^{\circ}$ \\
\hline & & L4-L5 & $\left(\begin{array}{l}38.4 \\
51.5\end{array}\right)$ & $\begin{array}{l}a=3.6 \\
b=3.3\end{array}$ & $13^{\circ}$ & $\left(\begin{array}{l}38.4 \\
50.7\end{array}\right)$ & $\begin{array}{l}a=2.3 \\
b=1.9\end{array}$ & $-35^{\circ}$ \\
\hline & & L5-SA & $\left(\begin{array}{l}31.3 \\
22.3\end{array}\right)$ & $\begin{array}{l}a=11.9 \\
b=7.9\end{array}$ & $-8^{\circ}$ & $\left(\begin{array}{l}33.2 \\
21.2\end{array}\right)$ & $\begin{array}{l}a=11.8 \\
b=7.9\end{array}$ & $2^{\circ}$ \\
\hline \multirow[t]{5}{*}{ PM } & \multirow[t]{5}{*}{$\begin{array}{l}35.1^{\circ} \pm 4.1^{\circ} \\
{\left[25.5^{\circ} \ldots 39.1^{\circ}\right]}\end{array}$} & L1-L2 & $\left(\begin{array}{c}29.7 \\
151.0\end{array}\right)$ & $\begin{array}{l}a=14.4 \\
b=2.1\end{array}$ & $7^{\circ}$ & $\left(\begin{array}{c}23.4 \\
151.6\end{array}\right)$ & $\begin{array}{l}a=37.1 \\
b=3.2\end{array}$ & $9^{\circ}$ \\
\hline & & L2-L3 & $\left(\begin{array}{c}35.1 \\
115.2\end{array}\right)$ & $\begin{array}{l}a=18.4 \\
b=2.2\end{array}$ & $13^{\circ}$ & $\left(\begin{array}{c}25.9 \\
113.6\end{array}\right)$ & $\begin{array}{l}a=42.2 \\
b=2.9\end{array}$ & $15^{\circ}$ \\
\hline & & L3-L4 & $\left(\begin{array}{l}39.3 \\
80.6\end{array}\right)$ & $\begin{array}{l}a=14.3 \\
b=2.9\end{array}$ & $24^{\circ}$ & $\left(\begin{array}{l}30.7 \\
77.1\end{array}\right)$ & $\begin{array}{l}a=26.2 \\
b=3.0\end{array}$ & $29^{\circ}$ \\
\hline & & L4-L5 & $\left(\begin{array}{l}38.6 \\
49.6\end{array}\right)$ & $\begin{array}{l}a=14.8 \\
b=4.0\end{array}$ & $32^{\circ}$ & $\left(\begin{array}{l}29.7 \\
44.0\end{array}\right)$ & $\begin{array}{l}a=24.7 \\
b=3.8\end{array}$ & $39^{\circ}$ \\
\hline & & L5-SA & $\left(\begin{array}{l}30.7 \\
22.5\end{array}\right)$ & $\begin{array}{l}a=18.3 \\
b=11.8\end{array}$ & $34^{\circ}$ & $\left(\begin{array}{l}22.5 \\
14.0\end{array}\right)$ & $\begin{array}{l}a=36.0 \\
b=7.9\end{array}$ & $40^{\circ}$ \\
\hline \multirow[t]{5}{*}{ PT } & \multirow[t]{5}{*}{$\begin{array}{l}37.2^{\circ} \pm 3.1^{\circ} \\
{\left[32.6^{\circ} \ldots 41.6^{\circ}\right]}\end{array}$} & L1-L2 & $\left(\begin{array}{c}31.0 \\
151.2\end{array}\right)$ & $\begin{array}{l}a=3.2 \\
b=1.9\end{array}$ & $-44^{\circ}$ & $\left(\begin{array}{c}30.1 \\
153.1\end{array}\right)$ & $\begin{array}{l}a=3.3 \\
b=2.3\end{array}$ & $-78^{\circ}$ \\
\hline & & L2-L3 & $\left(\begin{array}{c}36.9 \\
115.5\end{array}\right)$ & $\begin{array}{l}a=4.5 \\
b=1.9\end{array}$ & $-27^{\circ}$ & $\left(\begin{array}{c}34.7 \\
117.1\end{array}\right)$ & $\begin{array}{l}a=3.3 \\
b=2.7\end{array}$ & $-28^{\circ}$ \\
\hline & & L3-L4 & $\left(\begin{array}{l}40.8 \\
81.1\end{array}\right)$ & $\begin{array}{l}a=5.9 \\
b=2.3\end{array}$ & $-14^{\circ}$ & $\left(\begin{array}{l}37.8 \\
81.8\end{array}\right)$ & $\begin{array}{l}a=4.5 \\
b=2.9\end{array}$ & $16^{\circ}$ \\
\hline & & L4-L5 & $\left(\begin{array}{l}39.4 \\
50.1\end{array}\right)$ & $\begin{array}{l}a=6.8 \\
b=3.5\end{array}$ & $14^{\circ}$ & $\left(\begin{array}{l}35.9 \\
48.9\end{array}\right)$ & $\begin{array}{l}a=7.4 \\
b=2.7\end{array}$ & $35^{\circ}$ \\
\hline & & L5-SA & $\left(\begin{array}{l}29.4 \\
23.4\end{array}\right)$ & $\begin{array}{l}a=17.8 \\
b=10.6\end{array}$ & $-22^{\circ}$ & $\left(\begin{array}{l}28.5 \\
18.2\end{array}\right)$ & $\begin{array}{l}a=18.0 \\
b=8.6\end{array}$ & $39^{\circ}$ \\
\hline
\end{tabular}

Column two: range of motion (ROM) for the whole lumbar spine models. Columns 4-9: center points, first and second semi-axes lengths, as well as orientation angles for the non-weighted (columns 4-6) and weighted (columns 7-9) ellipses at each spinal level (L1-L2 to L5-SA) 
narrower than the non-weighted ellipses, indicating that the variation in the centrode's location took place well after the beginning of simulation. Due to the narrowness and the resulting indistinguishability of the two semi-axes, the orientation angles became a random number on the interval $-90^{\circ} \ldots+90^{\circ}$. ROM values of around $10.7^{\circ}$ were already mentioned to be comparable to the NPM scenario, but also matched the literature data of cadaver experiments (Heuer et al. 2007, fig. 4).

PM Fig. 3c shows the ICR-time courses that resulted from simulating the muscle-driven scenario with a preload of $500 \mathrm{~N}$ (PM) acting on L1 and along the gravity line, as described in Sect. 2.3. The different levels of the individual loaded lumbar spine models showed a comparable ICR-time courses as in the NPM mode, i.e., the ICR-time courses started inferior-posterior to the COM of the caudal vertebra and moved in a superior-anterior direction. Likewise, the lower the spine level, the more ellipse centers moved in superior-anterior direction, here with the center of the L2-L3 ellipse almost congruent to the L3 COM. However, in levels L1-L2 and L2-L3, few early ICR data points lay markedly posterior to the COM, thus forming a tail-shaped path toward the ellipse center. Consequently, the respective first semi-axis lengths of the weighted ellipses were longer than for the non-weighted ellipses, and for both cases longer in the PM than in the NPM scenario. Yet, the width of the ellipses (the second semi-axis lengths) showed no such differences and likewise increased in caudal direction, cf. Table 2. Ellipse centers for the PM scenario were found to be near to those from the NPM scenario. The orientation angle of the non-weighted and weighted ellipses increased in caudal direction from $7^{\circ}$ and $9^{\circ}$ to $33^{\circ}$ and $40^{\circ}$, respectively. The latter angles (L5-SA) were thus similar to the NPM scenario. The ROM ranged from $25.5^{\circ}$ to $39.1^{\circ}$ and was comparable to the ROM of the PT scenario. Furthermore, the ROM was about four times larger in the scenarios with than without preload.

PT Fig. 3d shows the ICR-time courses that resulted from simulating the torque-driven scenario with a preload of $500 \mathrm{~N}$ (PT). Comparable to the NPT scenario, each level of the PT models yielded similar centrodes, which were located in a narrow region, superior-anterior and close to the COM of the caudal vertebra. Here, the lengths of the first and second semi-axes were on average 1.5-2 times longer than in the NPT scenario, but still small compared to the muscle-driven scenarios. As in the NPT scenario, the range of quotients between the first and second semi-axis lengths was smaller in NPM $(1.4 \ldots 2.7)$ than in the muscle-driven scenarios $(1.5 \ldots 14)$. In addition, there was no noticeable difference between the semi-axis lengths of the weighted and non-weighted ellipses. Contrary to the muscle-driven scenarios, the orientation angles of the non-weighted ellipses were negative, except for the level L4-L5. The orientation angles of the weighted ellipses increased, as in the PM scenario, in a caudal direction from $-78^{\circ}$ to $39^{\circ}$. ROM values between $32.6^{\circ}$ and $41.6^{\circ}$ were comparable to the PM scenario. In both NPM and NPT, the size of the ellipses increases in caudal direction, indicating an amplified translation instead of pure rotation (the narrower the ellipse, the purer the rotation).

\section{Discussion}

\subsection{On torque-driven experiments and physiological insights}

Bending forward, reaching for a crate of beer and lifting it up incorrectly may result in high loading peaks within the lumbar spine (Nachemson 1965; Wilke et al. 1999). Due to unfavorable lever arms with regard to the joints, the gravitational forces of the body parts can cause high torques within the human body, which must be compensated for by the muscles. As a consequence, the internal muscle forces, transmitted according to Newton's law (actio=reactio), eventually generate high compressive and shear forces in the various spinal structures. When experimentally isolating certain structures (e.g. vertebrae, IVDs and ligaments) while leaving others out (e.g. muscles), experimental observations may greatly differ from physiological reality. For example, isolated (lumbar) spines had been observed to "buckle" under compressive loads not even close to in vivo magnitudes (Crisco 1989). To compensate for the missing supporting structures, when applying high loads on cadaver specimen, the concept of follower load was established (Patwardhan et al. 1999; Rohlmann et al. 2009a). At this, a guiding rail ought to ensure a purely compressive force transmission and prevent the occurrence of shear forces, which would lead to buckling. Consequently, the forces were applied "tangent to the spinal curve, passing through the center of rotation of each segment" (Patwardhan et al. 1999, fig. 1), which was supposed to be located perfectly in between the vertebrae. It was found that the path of the follower load influences the model output and should be optimized in a sense that it passes through the centers of rotation between vertebrae (Dreischarf et al. 2010).

In both in vitro experiment and follower-load model, torque had not been physiologically induced by muscle forces, but artificially "applied" by spine testers to induce a flexion movement. We herein revealed that this method differs substantially from muscle-driven movements by means of the corresponding centrodes: torque-driven centrodes, regardless the individual spine geometry, can be found in a narrow region superior-anterior to the caudal vertebra's COM. Muscle-driven centrodes show a more individual behavior and stretch over a wider range. These observations 
hold true for non-preload and preload scenarios alike, under our model assumptions. Of course, our presented model is far from capturing every physiological aspect of in vivo force transmission, since we omitted most of the whole body's structures. However, our approach may serve as a starting point for pursuing centrode-based investigations. For example, the herein introduced concept of confidence ellipses may be utilized to assess the influence of model parameter changes (sensitivities) on the centrode location. These investigations could include the influence of ligament stiffness or failure (Abouhossein et al. 2013; Alapan et al. 2013; Putzer et al. 2016), joint forces (Senteler et al. 2017), implant positioning (Dreischarf et al. 2015; Rohlmann et al. 2010) or variable load application (Rohlmann et al. 2009b).

On the one hand, our findings support the application of the follower load concept for recreating in vitro experiments: torque-induced centrodes (and thus the path of the follower load) are virtually inert to individual geometries, instants of time, or loading scenarios. Hence, once established, the follower load can remain unchanged during the whole simulation. On the other hand, our findings speak against the application of the follower load concept for recreating in vivo experiments: the ICR location is known to change during physiological motion (Aiyangar et al. 2017, fig. 2), as is likewise visible in our Fig. 3a, c. This change in ICR location indicates the existence of translational rather than purely rotational movements of vertebrae relative to each other, which cannot be captured while utilizing a follower load.

Summarizing, our findings suggest that modeled spinal motion have to be compared with caution regarding their impetus. When aiming for physiological insights, muscle-driven models ought to be utilized. Here, it might be worth investigating whether the method of muscle control, e.g. inverse dynamic (Happee 1994), EMG-driven (Lloyd and Besier 2003) or forward dynamic (Rupp et al. 2015), has significant influence on the respective centrodes. Likewise, incorporating muscle deflection on larger muscles (Hammer et al. 2019) might lead to altered centrodes. When aiming at recreating in vitro experiments that require stabilizing follower load, torque-driven models constitute a more natural choice, but their results cannot directly be transferred to physiological reality, as statements on possible medical implications (see the Introduction and the next Sect. 4.2) might not satisfy the expectation.

\subsection{Centrodes from a medical point of view}

The study of spinal motion is of utmost importance when aiming to understand the formation of disorders and the effects of surgical interventions. In clinical practice, hypermobile segments or degenerative structures commonly undergo fixing procedures. Stabilizing only one or few segments is hereby generally considered successful, although the individual spinal motion pattern is not examined in detail. If long constructs are required, or when aiming to preserve or restore "healthy" motion by application of dynamic implants (motion preserving implants), an exact balance of the resulting forces and thus profound knowledge of the motion pattern is, however, mandatory. Numerous research on motion patterns has been conducted on the cervical spine (Amevo et al. 1991; Anderst et al. 2015; Wachowski et al. 2017). This area is not only less complex than the lumbar area (due to less soft tissue involved), but also the region where most dynamic implants-especially disk prostheses-are used. The highest loads and consequently the location where degenerative changes occur first, is however be found the lumbar spine (Auerbach et al. 2008).

Changes in (lumbar) spinal kinematics have been observed following surgical procedures (spondylodesis using different techniques (Nomoto et al. 2019), facetectomy (Zeng et al. 2017), implantation of disk prothesis (Yue et al. 2019) or pedicle screw-based dynamic implants (Prud'homme et al. 2015)), but do also occur naturally due to degeneration or trauma (Amevo et al. 1992) as well as in obese patients (Rodriguez-Martinez et al. 2016). In addition, several studies, in vitro and in vivo, have been conducted to analyze lumbar spinal kinematics and to determine the centrode under healthy and degenerative conditions, see (Widmer et al. 2019) for a review. So far, however, there exists neither mechanistic nor statistical criteria linking the mere observation to a quantitative kinematic assessment, let alone to predicting the effects of surgical interventions. Given sufficient experimental data, the herein presented concept of confidence ellipses could help correlating centrodes to their corresponding clinical syndromes or medical treatments.

CT scans and bending fluoroscopy are generally available for most spinal patients. These image data allow for example to assess the grade of disk degeneration (Quint and Wilke 2008), but other structural properties-as the individual stiffness of certain ligaments or the strength of supporting muscles - can only be approximated. Further evaluation of medical image data, e.g. water-fat MRI (Schlaeger et al. 2018), could provide estimates for individualized muscle parameters, such as the maximum force. Further, stereo X-ray films (Aiyangar et al. 2017) could allow for a preciser tracing of the ICR location. Overall, the utilization of individual data constitutes an important step toward an individual spine model that could eventually be used to predict the effect of surgical interventions and to optimize operative plans before surgery (Kantelhardt et al. 2015). Implants and their positions could thus be selected, not solely but among others, on basis of individual centrode simulations, see also the next section. 


\subsection{Consecutive fixation systematically alters the centrode: an exemplary scenario}

Modeling load changes in lumbar spines, as a result of surgical implant placement, are utilized to assess the required medical procedure a priori (Xu et al. 2019). Additional criteria could be derived by classifying the respective centrode paths. In the literature, however, we identified two major issues in existing studies. First, lumbar spine models (Kiapour et al. 2012), which are validated using in vivo data (Pearcy and Bogduk 1988), where the center of rotation had been calculated only between the start and the endpoint of the movement, omit all mid-range dynamical information (Dombrowski et al. 2018). Second, models (Abouhossein et al. 2013), which are validated using torque-driven in vitro data (Rousseau et al. 2006), where one of the vertebrae had been fixed, obtain centrodes in between the vertebrae, which is contrary to in vivo findings (Aiyangar et al. 2017, fig. 2). These two issues can also be found combined (Naserkhaki et al. 2018; Schmidt et al. 2008).

In this section, we cursory glance at possible consequences of consecutively fixating spinal segments, i.e., inserting rigid implants starting from the sacrum and moving cranially up to L2. Figure 4 visualize the scenario and the results from a single NPT model that was utilized for a purely exemplary purpose, thus no error ellipses were calculated. Although we stated above that muscle-driven models ought to be consulted when aiming at confidable physiological findings, more sophisticated structures are required in our model to allow for significant ROM in the ultimately fixated scenario. Let SA- $X$ denote a fixation from the SA upward to segment $X$, i.e., SA-L2 refers to a spinal unit where SA to L2 are rigidly connected by implants. The ROM of the flexion induced by $10 \mathrm{Nm}$ expectedly decreased during consecutive fixation: from $\mathrm{ROM}_{\mathrm{SA}-\mathrm{SA}}=10.5^{\circ}$ to $\mathrm{ROM}_{\mathrm{SA}-\mathrm{L} 2}=0.3^{\circ}$. The corresponding centrodes showed a systematic behavior: the further away segments were from the fixation, the narrower and more inferior-posterior the centrodes were located, ultimately approaching the COM of the caudal body. Within these centrodes, the migration path of the ICR was found to evolve superior-anterior. The segments, which were located directly cranial to the fixation showed a hookshaped centrode right in the middle of the respective IVD.

These findings, although conducted with a rather simplified model, suggest a critical view on the aforementioned issues: mid-range dynamics as well as multi- $(>2$ )-body analyses could play a crucial role in assessing operational methods such as optimizing implant positioning (Haher et al. 1991; Niosi et al. 2006).
Fig. 4 a Two-dimensional centrode locations for five consecutive degrees of fixation (colored dots), obtained from running NPT scenarios on an individual lumbar spine model. The lateral view on the lumbar spine is shown in the background and crucial regions are highlighted by a zoom. b Semitransparent three-dimensional depiction of the corresponding implant (or pedicle screw) placements within the vertebral bodies, without further active or passive structures visible. Same colors indicate the same degree of fixation: (1) SA-SA (green, standard NPT as in Fig. 3b), (2) SA-L5 (violet), (3) SA-L4 (yellow), (4) SA-L3 (red), (5) SA-L2 (blue). ICR coordinates were computed as described in Sect. 2.2

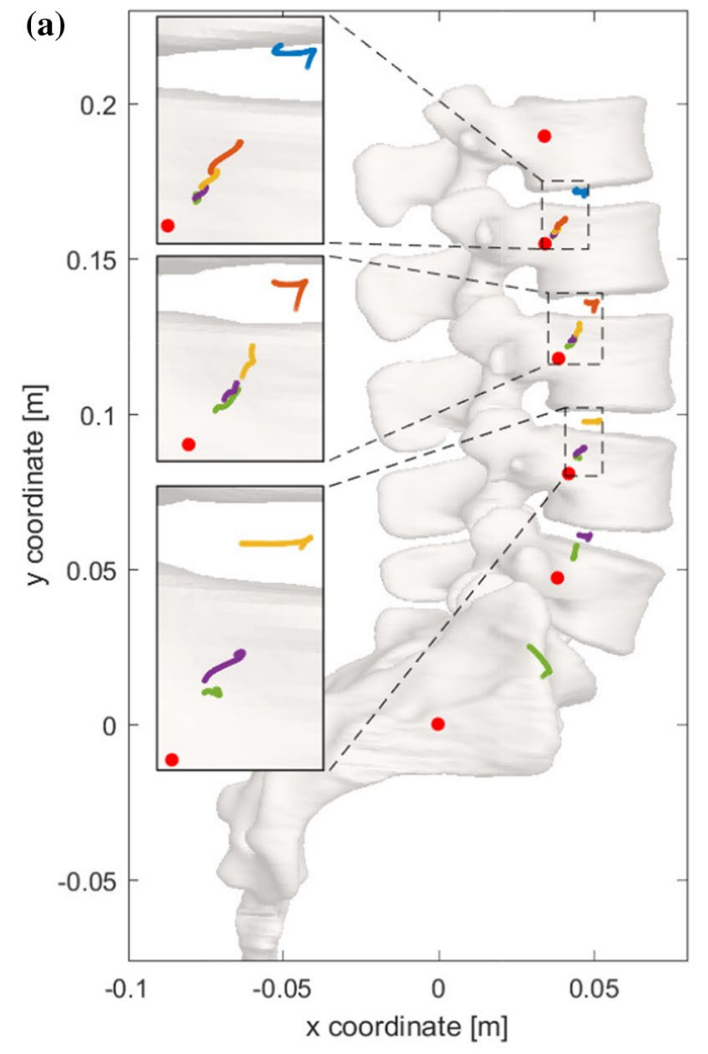

(b)

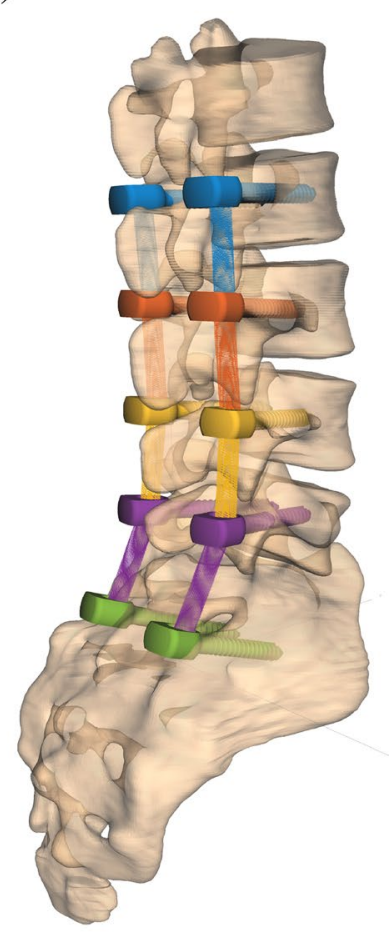




\section{Supplementary material}

Three video files showing an exemplary flexion movement, from neutral position to full bending (cf. Fig. 1a, d), are provided. Each video depicts a different view on the lumbar spine (frontal, lateral and lateral-frontal). At every time instance, the corresponding FHA are shown.

Author's Contribution RR developed the concept of the manuscript, wrote the core text and discussed physiological implications of the method. AM conducted the model calculations and discussed the effects of consecutive fixation. ND set up the model as well as the basic idea of this work. MK and SRK contributed equally on collecting image data, identifying ligament insertion points, and discussing the findings from a medical point of view. RF helped developing the calculation of the FHA and contributed geometrical interpretations of the ICR path locations. KG helped developing the model, provided the MBS graphics and conducted a final review of the manuscript.

Funding Open Access funding provided by Projekt DEAL. A.M. is supported by funding from Mechanical Systems Engineering Laboratory, EMPA-Swiss Federal Laboratories for Materials Science and Technology, Duebendorf, Switzerland.

\section{Compliance with ethical standards}

Conflict of interest The authors declare that they have no conflicts of interest.

Open Access This article is licensed under a Creative Commons Attribution 4.0 International License, which permits use, sharing, adaptation, distribution and reproduction in any medium or format, as long as you give appropriate credit to the original author(s) and the source, provide a link to the Creative Commons license, and indicate if changes were made. The images or other third party material in this article are included in the article's Creative Commons license, unless indicated otherwise in a credit line to the material. If material is not included in the article's Creative Commons license and your intended use is not permitted by statutory regulation or exceeds the permitted use, you will need to obtain permission directly from the copyright holder. To view a copy of this license, visit http://creativecommons.org/licenses/by/4.0/.

\section{References}

Abouhossein A, Weisse B, Ferguson SJ (2013) Quantifying the centre of rotation pattern in a multi-body model of the lumbar spine. Comput Methods Biomech Biomed Eng 16(12):1362-1373

Aiyangar A, Zheng L, Anderst WJ, Zhang X (2017) Instantaneous centers of rotation for lumbar segmental extension in vivo. J Biomech 52(1):113-121

Alapan Y, Demir C, Kaner T, Guclu R, Ingeoglu S (2013) Instantaneous center of rotation behavior of the lumbar spine with ligament failure. J Neurosurg Spine 18(6):617-626

Amevo B, Worth D, Bogduk N (1991) Instantaneous axes of rotation of the typical cervical motion segments: II Optimization of technical errors. Clin Biomech (Bristol, Avon) 6(1):38-46

Amevo B, Aprill C, Bogkuk N (1992) Abnormal instantaneous axes of rotation in patients with neck pain. Spine 17(7):748-756
Anderst WJ, Donaldson WF, Lee JY, Kang JD (2015) Three-dimensional intervertebral kinematics in the healthy young adult cervical spine during dynamic functional loading. J Biomech 48(7):1286-1293

Auerbach JD, Jones KJ, Fras CI, Balderston JR, Rushton SA, Chin KR (2008) The prevalence of indications and contraindications to cervical total disc replacement. Spine J 8(5):711-716

Baillargeon E, Anderst WJ (2013) Sensitivity, reliability and accuracy of the instant center of rotation calculation in the cervical spine during in vivo dynamic flexion-extension. J Biomech 46(4):670-676

Bogduk N, Amevo B, Pearcy M (1995) A biological basis for instantaneous centres of rotation of the vertebral column. Proc Inst Mech Eng H 209(3):177-183

Chazal J, Tanguy A, Bourges M, Gaurel G, Escande G, Guillot M, Vaneuville G (1985) Biomechanical properties of spinal ligaments and a histological study of the supraspinal ligament in traction. J Biomech 18(3):167-176

Christophy M, Faruk SN, Lotz JC, O'Reilly OM (2012) A musculoskeletal model for the lumbar spine. Biomech Model Mechanobiol 11(1-2):19-34

Cossette JW, Farfan HF, Robertson GH, Wells RV (1971) The instantaneous center of rotation of the third lumbar invertebral joint. J Biomech 4(2): 149-153

Crisco JJ (1989) The biomechanical stability of the human lumbar spine: experimental and theoretical investigations. $\mathrm{PhD}$ thesis, Yale University, New Haven, CT, USA

Damm N, Rockenfeller R, Gruber K (2019) Lumbar spine ligament characteristics extracted from stepwise reduction experiment allow for preciser modeling than literature data. Biomech Model Mechanobiol 19:893-910

Dombrowski ME, Rynearson B, LeVasseur C, Adgate Z, Donaldson WF, Lee JY, Aiyangar A, Anderst WJ (2018) Effect of graded facetectomy on biomechanics of dynesys dynamic stabilization system. Eur Spine J 27(4):752-762

Draper NR (1998) Applied regression analysis, vol 3. Wiley, New York

Dreischarf M, Zander T, Bergmann G, Rohlmann A (2010) A nonoptimized follower load path may cause considerable intervertebral rotations. J Biomech 43(13):2625-2628

Dreischarf M, Schmidt H, Putzier M, Zander T (2015) Biomechanics of the L5-S1 motion segment after total disc replacementinfluence of iatrogenic distraction, implant positioning and preoperative disc height on the range of motion and loading of facet joints. J Biomech 48(12):3283-3291

Ellingson AM, Nuckley DJ (2015) Altered helical axis patterns of the lumbar spine indicate increased instability with disc degeneration. J Biomech 48(2):361-369

Galton F (1886) Regression towards mediocrity in hereditary strature. J Anthropol Inst G B Irel 15(1):246-263

Gertzbein SD, Seligman J, Holtby R, Chan KH, Kapasouri A, Tile M, Cruickshank B (1985) Centrode patterns and segmental instability in degenerative disc disease. Spine 10(3):257-261

Guenther M, Schmitt S, Wank V (2007) High-frequency oscillations as a consequence of neglected serial damping in hill-type muscle models. Biol Cybern 97(1):63-79

Haeufle DFB, Guenther M, Bayer A, Schmitt S (2014) Hill-type muscle model with serial damping and eccentric force-velocity. J Biomech 47(6):1531-1536

Haher TR, Berman M, O’Brien M, Tallman Felmly W, Choueka J, Welin D, Chow G, Vassiliou A (1991) The effect of the three columns of the spine on the instantaneous axis of rotation in flexion and extension. Spine 16(Suppl. 8):S312-S318

Hammer M, Günther M, Haeufle DFB, Schmitt S (2019) Tailoring anatomical muscle paths: a sheath-like solution for muscle 
routing in musculoskeletal computer models. Math Biosci 311(1):68-81

Happee R (1994) Inverse dynamic optimization including muscular dynamics, a new simulation method applied to goal directed movements. J Biomech 27(7):953-960

Heuer F, Schmidt H, Klezl Z, Claes L, Wilke HJ (2007) Stepwise reduction of functional spinal structures increase range of motion and change lordosis angle. J Biomech 40(2):271-280

Kantelhardt SR, Hausen U, Kosterhon M, Amr AN, Gruber K, Giese A (2015) Computer simulation and image guidance for individualised dynamic spinal stabilization. Int J Comput Assist Radiol Surg 10(8):1325-1332

Kiapour A, Ambati D, Hoy R, Goel VK (2012) Effect of graded facetectomy on biomechanics of dynesys dynamic stabilization system. Spine 37(10):E581-E589

Kwon Y (2008) Measurement for deriving kinematic parameters: numerical methods. In: Hong Y, Bartlett R (eds) Routledge handbook of biomechanics and human movement science, chap 12, Routledge, London, pp 156-181

Le Huec JC, Saddiki R, Franke J, Rigal JB, Aunoble S (2011) Equilibrium of the human body and the gravity line: the basics. Eur Spine J 20(Suppl. 5):558-563

Lloyd DG, Besier TF (2003) An EMG-driven musculoskeletal model to estimate muscle forces and knee joint moments in vivo. J Biomech 36(6):765-776

Mörl F, Siebert T, Haeufle DFB (2015) Contraction dynamics and function of the muscle-tendon complex depend on the muscle fibre-tendon length ratio: a simulation study. Biomech Model Mechanobiol 15(1):245-258

Mörl F, Günther M, Riede JM, Hammer M, Schmitt S (2020) Loads distributed in vivo among vertebrae, muscles, spinal ligaments, and intervertebral discs in a passively flexed lumbar spine. Biomech Model Mechanobiol. https://doi.org/10.1007/s10237-02001322-7

Nachemson A (1965) The effect of forward leaning on lumbar intradiscal pressure. Acta Physiol Scand 35(1-4):314-328

Naserkhaki S, Arjmand N, Shirazi-Adl A, Farahmand F, El-Rich M (2018) Effects of eight different ligament property datasets on biomechanics of a lumbar 14-15 finite element model. J Biomech 70(1):33-42

Niosi CA, Zhu QA, Wilson DC, Keynan O, Wilson DR, Oxland TR (2006) Biomechanical characterization of the three-dimensional kinematic behaviour of the dynesys dynamic stabilization system: an in vitro study. Eur Spine J 15(6):913-922

Nomoto EK, Fogel GR, Rasouli A, Bundy JV, Turner AW (2019) Biomechanical analysis of cortical versus pedicle screw fixation stability in TLIF, PLIF, and XLIF applications. Glob Spine J 9(2):162-168

Ogston NG, King GH, Gertzbein SD, Tile M, Kapasouri A, Rubenstein JD (1986) Centrode patterns in the lumbar spine. Baseline studies in normal subjects. Spine 11(6):591-595

Patwardhan AG, Havey RM, Meade KP, Lee B, Dunlap B (1999) A follower load increases the load-carrying capacity of the lumbar spine in compression. Spine 24(10):1003-1009

Pearcy MJ, Bogduk N (1988) Instantaneous axes of rotation of the lumbar intervertebral joints. Spine 13(9):1033-1041

Prud'homme M, Barrios C, Rouch P, Charles YP, Steib JP, Skalli W (2015) Clinical outcomes and complications after pedicleanchored dynamic or hybrid lumbar spine stabilization: a systematic literature review. J Spinal Disord Tech 28(8):E439-E448

Putzer M, Auer S, Malpica W, Suess F, Dendorfer S (2016) A numerical study to determine the effect of ligament stiffness on kinematics of the lumbar spine during flexion. BMC Musculoskelet Disord 17:95
Qiu TX, Teo EC, Lee KK, Ng HW, Yang K (2003) Validation of T10 T11 finite element model and determination of instantaneous axes of rotations in three anatomical planes. Spine 28(24):2694-2699

Quint U, Wilke HJ (2008) Grading of degenerative disk disease and functional impairment: imaging versus patho-anatomical findings. Eur Spine J 17(12):1705-1713

Reuleaux F (1875) Theoretische kinematik. Friedrich Vieweg und Sohn, Braunschweig

Rockenfeller R, Guenther M (2016) Extracting low-velocity concentric and eccentric dynamic muscle properties from isometric contraction experiments. Math Biosci 278(1):77-93

Rockenfeller R, Guenther M (2018) Inter-filament spacing mediates calcium binding to troponin: a simple geometric-mechanistic model explains the shift of force-length maxima with muscle activation. J Theor Biol 454(1):240-252

Rodriguez-Martinez NG, Perrez-Orribo L, Kalb S, Reyes PM, Newcomb AG, Hughes J, Theodore N, Crawford NR (2016) The role of obesity in the biomechanics and radiological changes of the spine: an in vitro study. J Neurosurg Spine 24(4):615-623

Rohlmann A, Zander T, Rao M, Bergmann G (2009a) Applying a follower load delivers realistic results for simulating standing. J Biomech 42(10): 1520-1526

Rohlmann A, Zander T, Rao M, Bergmann G (2009b) Realistic loading conditions for upper body bending. J Biomech 42(7):884-890

Rohlmann A, Boustani HN, Bergmann G, Zander T (2010) Effect of a pedicle-screw-based motion preservation system on lumbar spine biomechanics: a probabilistic finite element study with subsequent sensitivity analysis. J Biomech 43(15):2963-2969

Rousseau MA, Bradford DS, Hadi TM, Pedersen KL, Lotz JC (2006) The instant axis of rotation influences facet forces at L5/S1 during flexion/extension and lateral bending. Eur Spine J 15(3):299-307

Rupp TK, Ehlers W, Karajan N, Günther M, Schmitt S (2015) A forward dynamics simulation of human lumbar spine flexion predicting the load sharing of intervertebral discs, ligaments, and muscles. Biomech Model Mechanobiol 14(5):1081-1105

Schlaeger S, Freitag F, Klupp E, Dieckmeyer M, Weidlich D, Inhuber S, Deschauer M, Schoser B, Bublitz S, Montagnese F, Zimmer C, Rummeny EJ, Karampinos DC, Kirschke JS, Baum T (2018) Thigh muscle segmentation of chemical shift encoding-based water-fat magnetic resonance images: the reference database MyoSegmenTUM. PLoS ONE 13(6):e0198200

Schmidt H, Heuer F, Claes L, Wilke HJ (2008) The relation between the instantaneous center of rotation and facet joint forces: a finite element analysis. Clin Biomech 23(3):270-278

Schünke M, Schulte E, Schumacher U, Voll M, Wesker K (2015) Prometheus Lernatlas der Anatomie. Thieme, Stuttgart

Senteler M, Aiyangar A, Weisse B, Farshad M, Snedeker JG (2017) Sensitivity of intervertebral joint forces to center of rotation location and trends along its migration path. J Biomech 70(1):140-148

Shirazi-Adl A, Ahmed AM, Shrivastava SC (1986) Mechanical response of a lumbar motion segment in axial torque alone and combined with compression. Spine 11(9):914-927

Spoor CW, Veldpaus FE (1980) Rigid body motion calculated from spatial co-ordinates of markers. J Biomech 13(4):391-393

Spruyt V (2014) How to draw an error ellipse representing the covariance matrix? https://www.visiondummy.com/2014/04/draw-error -ellipse-representing-covariance-matrix. Last Accessed July 2019

Wachowski MM, Weiland J, Wagner M, Gezzi R, Kubein-Meesenburg D, Nägerl H (2017) Kinematics of cervical segments C5/C6 in axial rotation before and after total disc arthroplasty. Eur Spine J 26(9):2425-2433

White AA, Panjabi MM (1990) Clinical biomechanics of the spine, vol 2. JB Lippincott Company, Philadelphia

Widmer J, Fornaciari P, Senteler M, Roth T, Snedeker JG, Farshad M (2019) Kinematics of the spine under healthy and 
degenerative conditions: a systematic review. Ann Biomed Eng 47(7):1491-1522

Wilke HJ, Wolf S, Claes LE, Arand M, Wiesend A (1996) Influence of varying muscle forces on lumbar intradiscal pressure: an in vitro study. J Biomech 29(4):549-555

Wilke HJ, Neef P, Caimi M, Hoogland T, Claes LE (1999) New in vivo measurements of pressures in the intervertebral disc in daily life. Spine 24(8):755-762

Xu M, Yang J, Liebermann I, Haddas R (2019) Stress distribution in vertebral bone and pedicle screw and screw-bone load transfers among various fixation methods for lumbar spine surgical: A finite element study. Med Eng Phys 63(1):26-32

Yoshioka T, Tsuji H, Hirano N, Sainoh S (1990) Motion characteristic of the normal lumbar spine in young adults: instantaneous axis of rotation and vertebral center motion analyses. J Spinal Disord 3(2):103-113

Yue JJ, Garcia R, Blumenthal S, Coric D, Patel VV, Dinh DH, Buttermann GR, Deutsch H, Miller LE, Persaud EJ, Ferko NC (2019)
Five-year results of a randomized controlled trial for lumbar artificial discs in single-level degenerative disc disease. Spine (Phila $\mathrm{Pa}$ 1976) 35(5):772-779

Zeng ZL, Zhu R, Wu YC, Zuo W, Yu Y, Wang JJ, Cheng LM (2017) Effect of graded facetectomy on lumbar biomechanics. J Healthc Eng. https://doi.org/10.1155/2017/7981513

Zwambag DP, Brown SHM (2020) Experimental validation of a novel spine model demonstrates the large contribution of passive muscle to the flexion relaxation phenomenon. J Biomech 102(1):109431

Publisher's Note Springer Nature remains neutral with regard to jurisdictional claims in published maps and institutional affiliations. 